\title{
Evaluation of the Mooney Viscosity of Natural Rubber by Near- Infrared Spectroscopy
}

\author{
Celio Pasquini and Fernanda Crivelari Figueiredo \\ Instituto de Química, Universidade Estadual de Campinas, \\ Campinas SP, Brazil \\ Benildes Prince \\ BRASLATEX, Indústria de Comércio de Borrachas Ltda., \\ Bálsamo SP, Brazil
}

\begin{abstract}
The direct near-infrared spectroscopic reflectance measurements of prevulcanized natural rubber (brown crepe) was employed for determination of its Mooney viscosity. NIR reflectance spectra were obtained for a total of 100 samples whose Mooney viscosity (VM in the range 68-95 units) have been determined by the standard reference procedure using a commercial computerized Mooney viscometer. These samples were employed as the raw material or were treated to achieve better homogenization. A Fourier transform near infrared (FT-NIR) spectrophotometer was employed, and the reflectance spectra were obtained with resolution of $4 \mathrm{~cm}^{-1}$ in the range $4000-10,000 \mathrm{~cm}^{-1}$ as an average of 75 scans. The samples were split in a calibration set containing 70 samples and in an external validation set consisting of the remaining 30 samples. The calibration and validation spectra sets were treated to correct for baseline shift, further transformed by first derivative and finally modeled by partial least squares (PLS) employing four latent variables. The model was evaluated with the external sample test set, and a RMSEP of 3.6 and 3.9 units of Mooney viscosity were obtained for homogenized and nonhomogenized samples,

Received 27 November 2004, Accepted 18 March 2005

This paper was by special invitation as a contribution to a special issue of the journal entitled "Quantitative Vibrational Spectrometry in the 21st Century." This special issue was organized by Professor Miguel de la Guardia, Professor of Analytical Chemistry at Valencia University, Spain.

Address correspondence to Celio Pasquini, Instituto de Química, Universidade Estadual de Campinas, Caixa Postal 6154, CEP 13083-862, Campinas SP, Brazil. E-mail: pasquini@iqm.unicamp.br
\end{abstract}


respectively. The NIR method is capable of determining the Mooney viscosity in few minutes in the non-pretreated sample with an error that is satisfactory for quality control of natural rubber destined for automobile tire manufacturing.

Keywords: Mooney viscosity, natural rubber, NIR spectroscopy, reflectance spectroscopy

\section{INTRODUCTION}

The elastic properties of natural rubber derive mainly from the characteristics of the polymeric isoprene in its cis isomer (cis-1,4-polyisoprene) whose structure is shown in the inset of Fig. 1. The less symmetrical structure of this isomer makes crystallization difficult and is responsible for the amorphous, flexible characteristics of the natural product.

Natural rubber has found an enormous number of applications in industrial products; notable among these is its use for making automobile tires. The natural prevulcanized latex (brown crepe) is evaluated for its quality and adequacy for various end uses. The Mooney viscosity is one of the more relevant parameters considering the destination of the natural rubber for tire production. Mooney viscosity is defined as the shearing torque resisting rotation of a cylindrical metal disk (or rotor) embedded in rubber within a cylindrical cavity. The dimensions of the shearing disk viscometer, test temperatures, and procedures for determining Mooney viscosity are

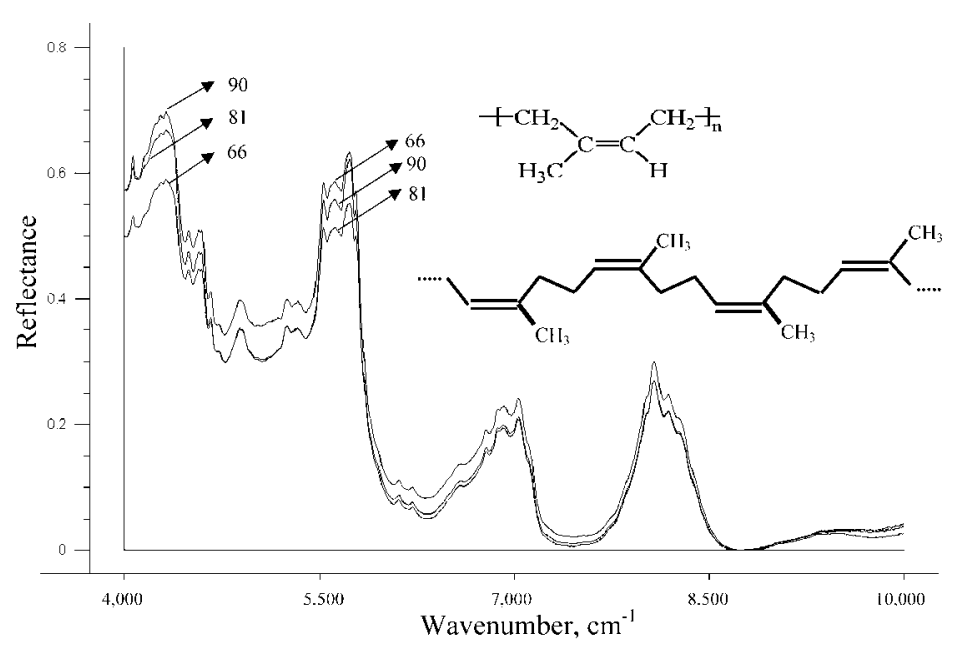

Figure 1. Three selected reflectance spectra of raw samples, after baseline offset correction, presenting Mooney viscosities in the range investigated in this work. The numbers represent the approximate Mooney viscosity of each sample. Inset: Chemical structure of the natural rubber: cis-1,4-polyisoprene. 
defined by the ASTM test method D1646-04. ${ }^{[1]}$ This parameter is measured in a heavy and complex mechanical instrument at high temperature $\left(100^{\circ} \mathrm{C}\right)$ where the resistance of a material to flow under stress is accessed by rotating a metallic disk on a rubber sample and measuring the torque necessary to keep the rotation at 2 revolutions per minute after a fixed time interval (5 to $10 \mathrm{~min}$ ).

Prevulcanized natural rubber presents Mooney viscosities in the range 60-90 and the acceptable range for use in tire manufacture is between 75 and 80 . The Mooney viscometer is an instrument specially constructed to determine the viscosity of the natural rubber. Computerized instruments can obtain the viscosity measurement with repeatability within 3 units although the mean standard deviation for an average value of the same piece of natural rubber can reach about 5 units. This last fact is due to the heterogeneous characteristics of the natural prevulcanized rubber. The measurement is time consuming (about $20 \mathrm{~min}$ for a single measurement), and the instrument requires constant maintenance. Furthermore, the sample needs to be homogenized by being preprocessed with a heated cylinder, an operation that requires an additional $15 \mathrm{~min}$.

The Mooney viscosity can be associated with the molar mass of the polymer chain. The average molar mass obtained by gel permeation chromatography (GPC) has been proposed for determination or has been associated with the Mooney viscosity. ${ }^{[2,3]}$ However, the proposed method requires the use of a GPC instrument and is another time-consuming technique that prevents rapid, in situ determination of the Mooney viscosity.

Near-infrared (NIR) spectroscopy has been used to determine bulk properties of a variety of samples, including artificial polymers. The information present in a NIR spectrum can be related to crystallinity,${ }^{[4]}$ molar mass, ${ }^{[2-5]}$ and viscosity ${ }^{[6-10]}$ of polymeric materials. However, the use of NIR spectroscopy for determination of Mooney viscosity of natural rubber has not yet been described in the literature.

This work aimed at the development and evaluation of the use of direct NIR reflectance spectral measurements for determination of the Mooney viscosity of natural rubber.

\section{EXPERIMENTAL}

\section{Instrumentation}

Mooney viscosity measurements were obtained in a computerized Mooney viscometer MV 2100 (Qualitest Inc., FL, USA). The results were employed for multivariate calibration and for evaluation of the accuracy of the model produced by the NIR method.

A Bomem model BM-164 FT-NIR spectrophotometer was employed to obtain the reflectance spectra of the natural rubber samples by using a 
Powder Samplir reflectance accessory. The spectra were registered in the range $4000-10,000 \mathrm{~cm}^{-1}$ as average of 75 scans with resolution of $4 \mathrm{~cm}^{-1}$. The total time interval necessary for spectrum acquisition was $40 \mathrm{~s}$.

\section{Samples and Sample Measurement}

One hundred samples of prevulcanized natural rubber (brown crepe) were collected from different lots during 1 month and cut into a block shape of about $1.5 \times 5 \times 3 \mathrm{~cm}$ in thickness, length, and width, respectively. Pieces of the same samples were processed with a heated cylinder to achieve better homogeneity. After processing, the sample presents the form of a thin sheet (about 2-mm thick). Pieces of these sheets, having dimensions of $3 \times 6 \mathrm{~cm}$, were also measured by reflectance.

The Mooney viscosity of the sample set was measured by the standard method and the values were in the range 65-95 units. The samples were divided in two subsets, one for calibration, containing 70 samples, and the other for external validation, containing the remaining 30 samples. The Mooney viscosity average values and their standard deviations were $(80.7 \pm 5.1)$ and $(81.6 \pm 4.6)$ Mooney units for the calibration and validation sets, respectively.

Natural rubber samples had their NIR reflectance spectra measured as the average of eight original spectra taken for each sheet (pretreated material) or block (raw material) (four measurements on each side sampled at equidistant points across the length of the sample). The average spectrum was saved for further processing.

\section{Computer and Software Facilities}

Spectral data were treated using an IBM-PC Pentium V $(1.6 \mathrm{GHz})$ compatible microcomputer running under a Windows Millennium operating system. The UNSCRAMBLER 9.1 chemometric software package was employed to perform the data pretreatment of the spectral data and for developing the partial least squares (PLS) models for Mooney viscosity.

\section{RESULTS AND DISCUSSION}

Figure 1 shows three spectra for raw samples of brown crepe of natural rubber with values of Mooney viscosity that are representative of the range observed for the sample set. The spectra are dominated by the strong absorptions of $-\mathrm{CH},-\mathrm{CH}_{2}$, and $-\mathrm{CH}_{3}$. However, some specific spectroscopic features can be attributed to the hydrogen atom bonded to an $\mathrm{R}_{2} \mathrm{C}=\mathrm{CHR}$, such as the peak located in the region between 4500 and $4350 \mathrm{~cm}^{-1}$. ${ }^{[11]}$ An observation 
of the natural isoprene polymer structure, shown in the inset of Fig. 1, reveals that the number of such groups can be promptly associated with the length of the isoprene chain. Furthermore, considering the $-\mathrm{CH}_{3}$ groups, the increase of absorbance between 8300 and $5900 \mathrm{~cm}^{-1}$, for instance, should reflect a decrease in the polymer chain length because shortening the chain will introduce more $-\mathrm{CH}_{3}$ terminal groups. However, it is not possible to observe a direct correlation between the intensities of absorbance and the value of the Mooney viscosity across the full spectrum. Obviously, many other complex spectrochemical features related to the overall behavior of the NIR spectrum in function of the average length of the polymer chains must be superimposed on the basic features.

The spectra sets for the raw and processed samples were evaluated by principal component analysis (PCA), and the scores distribution revealed that it was not possible to identify any outliers in the data set from such a score distribution. Also, the observation of the residuals versus leverage plot does not reveal any outliers regarding the spectral variables.

Following this preliminary multivariate qualitative evaluation, the set of 70 randomly selected spectra was employed to construct a PLS regression model to predict the Mooney viscosity. Internal full cross-validation was employed in the modeling stage. Unfortunately, as in most of the situations dealing with natural samples, the range of the property of interest and its distribution is far from being ideal. In fact, most of the values are centered in a narrow range of Mooney viscosities with only a few samples presenting values near the limits of the range.

The model produced by the original spectral set (70 samples) gives root mean square error of prediction (RMSEP) for internal full cross-validation of 3.6 units of Mooney viscosity and equal to 4.0 units for the external test set (30 samples). The reflectance spectrum of the natural rubber is clearly affected by scattering of the incident radiation on the rough surface of the brown crepe or of the processed product. Together with the natural sample heterogeneity, scattering causes a large fluctuation in the spectra baseline and relative absorption intensities, even for samples collected from the same rubber batch. Therefore, it was decided to try a pretreatment of the total spectra set by the multiplicative scattering correction (MSC) algorithm. ${ }^{[12]}$ However, the results were not improved significantly. The best results were obtained for the spectral set after baseline offset correction followed by taking the Savinstky-Golay first derivative (second-order polynomial, 10-point window). Figure 2 shows the complete data set for the raw samples after these transformations. Some tentative restrictions on spectral range and number of variables resulted in models that were not better than the one produced by using the total spectral range (4000 to $10,000 \mathrm{~cm}^{-1}$ ).

Results for the calibration stage show that four latent variables are required for the model to predict the Mooney viscosity. The RMSEP for the full cross-validation is 3.0 units of Mooney viscosity. The correlation coefficient and slope found between the predicted and reference values are 0.761 


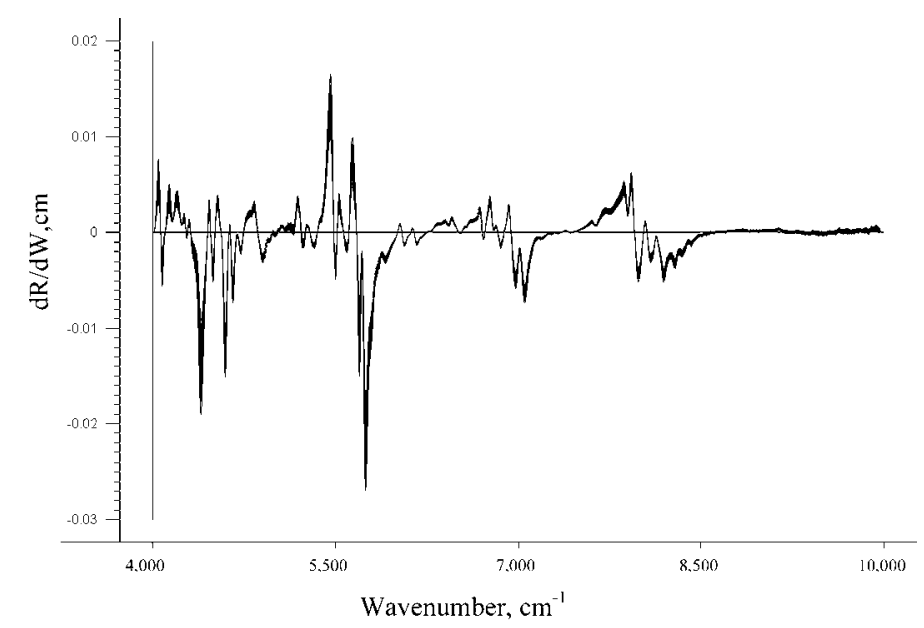

Figure 2. Complete set of reflectance spectra for 70 samples after baseline offset correction followed by the first derivative employed for the regression modeling.

and 0.615 , respectively. Figure 3 shows the results for the external validation (30 samples) of the PLS model. The RMSEP for the external validation is 3.9 units and the correlation coefficient is 0.633 , and the slope between the predicted and reference values is 0.580 .

The spectra set obtained for the same samples after processing (homogenization by pressing with a cylinder) shows lower fluctuation in

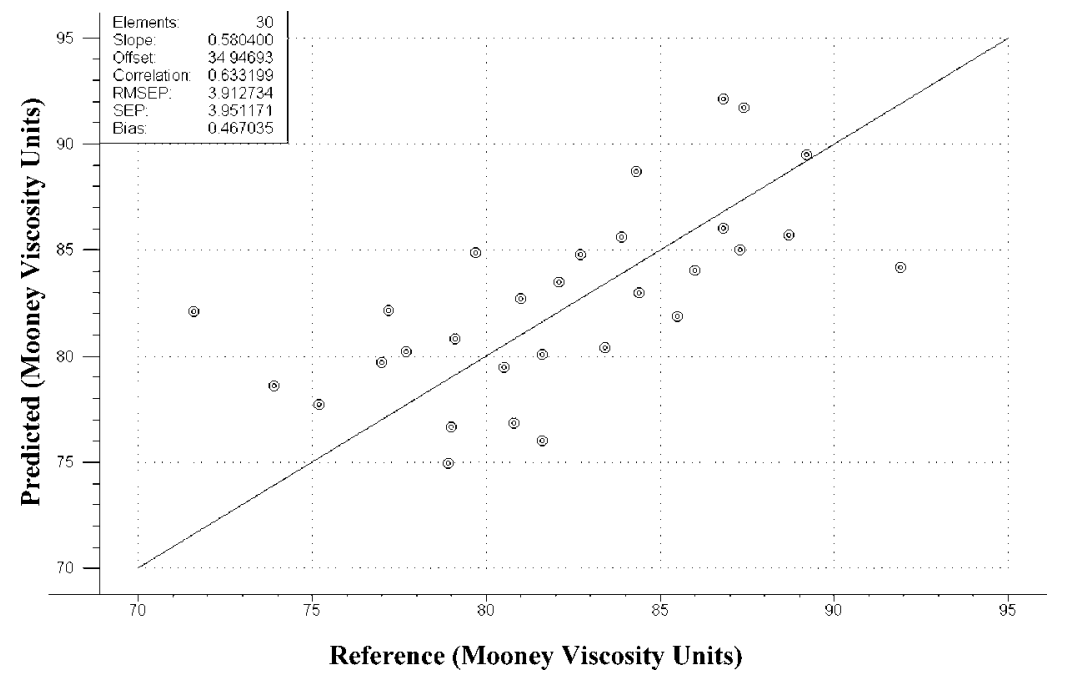

Figure 3. Summary of the external validation of the PLS model for prediction of Mooney viscosity based on NIR reflectance spectral data. 
terms of baseline and relative absorbances. They have been transformed in the same way as the spectra of the raw samples, and a PLS model was constructed. However, the results do not show any significant improvement over those obtained with the nontreated samples, showing a RMSEP for the external validation equal to 3.6 Mooney units.

The values obtained for the RMSEP agree with the repeatability of the Mooney viscometer and are adequate for use in routine determinations, considering the natural variability of the viscosity found for the same batch of natural rubber (about 5 units of Mooney viscosity).

The repeatability of the NIR reflectance method has been determined by using five different pieces of the same three rubber batches. The average values and standard deviation were $69 \pm 2 ; 76 \pm 4$; and $81 \pm 4$. Again, the repeatability of the NIR method is comparable with the standard procedure and mainly determined by the heterogeneity of the rubber sample.

The evaluation of the NIR reflectance spectroscopy in the determination of the Mooney viscosity of natural rubber showed promising results. The models developed in the current work suffer from the effects of the short range and a nonideal values distribution in the calibration and validation sample sets. In addition, the determination of the Mooney viscosity by the reference method is subjected to a precision that can reach about 5 units of viscosity. This reproducibility is similar to the standard deviation of the values of the viscosity of the samples employed in this work. Therefore, it would be very difficult to improve the results regarding the correlation coefficients and slopes for the internal and external validation made by employing the current data set.

On the other hand, the NIR reflectance method, due to its well-known characteristics of expeditiousness and direct measurement in the intact sample, provides a possible way for the on-line quality control of the rubber product.

\section{ACKNOWLEDGMENTS}

The authors are grateful to Dr. Carol H. Collins for manuscript revision. FCF is grateful to FAPESP for a fellowship (Proc. \#03/00147-0).

\section{REFERENCES}

1. ASTM D1646-04 Standard Test Methods for Rubber-Viscosity, Stress Relaxation, and nPre-Vulcanization Characteristics (Mooney Viscometer). ASTM International, USA, 2004.

2. Kramer, O.; Good, W. R. J. Correlating Mooney viscosity to average molecularweight. Appl. Polym. Science. 1972, 16, 2677-2684. 
3. Abdel-Goad, M.; Pyckhout-Hintzen, W.; Stefan Kahlel; Allgaier, J.; Richter, D.; Fetters, L. J. Rheological properties of 1,4-polyisoprene over a large molecular weight range. Macromolecules 2004, 37, 8135-8144.

4. Camacho, W.; Karlsson, S. J. Simultaneous determination of molecular weight and crystallinity of recycled HDPE by infrared spectroscopy and multivariate calibration. Appl. Polym. Sci. 2002, 85, 321-327.

5. Cherfi, A.; Fevotte, G.; Novat, C. J. Robust on-line measurement of conversion and molecular weight using NIR spectroscopy during solution polymerization. Appl. Polym. Sci. 2002, 85, 2510-2520.

6. Shenton, M. J.; Herman, H.; Stevens, G. C. Using spectroscopy with chemometrics to measure polymer molar mass. Polym. Int. 2000, 49, 1007-1013.

7. Chu, Z.; Hieftje, G. M. Near-infrared analysis of chemical-constituents and physical characteristics of polymers. Appl. Spectrosc. 1992, 46, 69-72.

8. Vanuum, M. P. B.; Lammers, H.; Dekleinj, J. P. Process analysis-properties of ply(ethylene-terephthalate) measured by near-infrared spectroscopy. 1. At-line analysis of poly(ethylene-terephthalate) chips. Macromol. Chem. Phys. 1995, 196, 2023-2028.

9. Lammers, H.; Vanuum, M. P. B.; Dekleinj, J. P. Process analysis-properties of ply(ethylene-terephthalate) measured by near-infrared spectroscopy. 2. In-line analysis of poly(ethylene-terephthalate) melt. Macromol. Chem. Phys. 1995, 196, 2029-2034.

10. Vedula, S.; Hansen, M. G. J. In-line fiber-optic near-infrared spectroscopy: monitoring of rheological properties in an extrusion process. Part II. Appl. Polym. Sci. 1998, 68, 873-889.

11. Williams, P.; Norris, K.; eds. Near-Infrared Technology, 2nd ed.; American Association of Cereal Chemistry, Inc.: St. Paul, MN, 2001.

12. Esbensen, K. Multivariate Data Analysis-In Pratice, 5th ed.; Camo Process AS: Oslo, 2002. 
Copyright of Spectroscopy Letters is the property of Taylor \& Francis Ltd. The copyright in an individual article may be maintained by the author in certain cases. Content may not be copied or emailed to multiple sites or posted to a listserv without the copyright holder's express written permission. However, users may print, download, or email articles for individual use. 